\title{
The role of electron microscopy in kidney lesions: A review of its diagnostic importance
}

\author{
Pant $\mathrm{AD}^{1}$, Solez $\mathrm{K}^{2}$ \\ ${ }^{1}$ Lecturer, Department of Pathology, Tribhuvan University Teaching Hospital, Kathmandu, Nepal \\ ${ }^{2}$ Professor of Pathology, Department of Laboratory Medicine and Pathology, University of Alberta, Canada
}

\author{
Keywords: \\ Biopsy; \\ Electron microscopy; \\ Glomerulonephritis; \\ Kidney; \\ Transplant glomerulopathy
}

\begin{abstract}
Electron microscopy is a technology which was at one time widely used for renal as well as non-renal benign and malignant diseases, but its use has been rapidly declining as hospitals all over the world cut down on expenses. This leaves the renal pathologist with only light microscopy and immunofluorescence at his disposal to diagnose diseases. Few studies have stated the importance of electron microscopy in routine renal biopsy reporting. We look at different cases where electron microscopy has been helpful in diagnosis and review the literature to assess the role this investigative modality still has to play in modern renal pathology.
\end{abstract}

\section{INTRODUCTION}

Now a days the only organ for which routine electron microscopy (EM) is performed is the kidney. It allows for detailed evaluation of cellular and extracellular contents in different parts of the kidney tissue and readily helps detect abnormalities which may not be apparent at light microscopic level. ${ }^{1}$ It was also used for diagnosing some musculoskeletal disorders and tumors, but its use in these tissues has rapidly declined due to the growth of immunohistochemisty. 2,3

Transmission electron microscopy has been a fundamental part of diagnosing renal lesions since its inception. Together with light microscopy (LM) and immunohistology, it forms the ideal triad of investigations for renal biopsy interpretations.

\section{Correspondence:}

Dr. Anil Dev Pant, $M D$

Department of Pathology, T.U.Teaching Hospital, Maharajgunj, Kathmandu, Nepal.E-mail:adpant@yahoo.com

\section{SPECIMEN HANDLING}

Received samples are usually immersed in cold physiological saline and cut into 3 pieces while viewing under a stereomicroscope; one for paraffin sections for light microscopy, one for frozen section for immunofluorescence, and one for EM. The EM specimen is fixed in 3\% glutaraldehyde, post-stained with osmium tetra-oxide, and embedded in epon. Thin sections are then cut with diamond knives and subsequently stained on grids, after which they are finally examined with the electron microscope. ${ }^{4}$

Even though EM may not be performed on all biopsies, it is a good idea to store tissue for it, in case the need arises. Another instance where this proves useful is when there are no glomeruli in the sample separated for immunofluorescence. Electron microscopy may also be done on paraffin blocks by re-embedding the biopsies into epon, in such cases where adequate tissue is not present in the sample separated for it. ${ }^{4}$ 


\section{ROLE OF ELECTRON MICROSCOPY IN RENAL BIOPSY}

The role of EM for part of biopsy evaluation had been evaluated in the late 1960s and early 1970s. It was found to have substantially different diagnoses in few of the cases and to provide additional important information in many others. $^{5}$

More specifically, EM shows details of cellular and extracellular components of glomeruli, including thickness, contour and integrity of basement membrane and mesangial matrix. Electron dense deposits and fibrillary deposits can be detected and distinguished in their respective areas, including sub-epithelial, intramembranous, and mesangial locations. Effacement of foot processes and endothelial cell swelling are also readily visible. Though few diseases require EM, many are made more readily apparent with the help of EM, and sometimes retrospectively after reassessment by light microscopy. Typical findings like thinning of glomerular basement membranes in various disorders, tubuloreticular inclusions and fingerprinting in lupus, fibrils of fibrillary glomerulonephritis and amyloidosis, and zebra bodies of Fabry's disease are seen on electron microscopy. ${ }^{1}$

Electron microscopy may help to correct an erroneous diagnosis made with only light microscopy and immunofluorescence. In cases where immunofluorescence samples show no glomeruli, EM can play an important role in the diagnosis, which may sparea re-biopsy for the patient. $^{6}$

Unfortunately, EM is an investigation that requires high maintenance and is labor intensive for technical staff. It also requires a large operational space and is costly to purchase and maintain.Hence, tissue has been further diminishing as hospitals worldwide cut down on expenditure in these unfavorable economic times. It is indeed ironic to see that educational institutions are actually taking this more pragmatic and less academic step as they cut down on costs. ${ }^{5,7}$

An study performed in the U.K. surveyed several hospitals with electron microscopes and found that they would eventually be closing and outsourcing their material to other institutions. ${ }^{8}$ In addition to the previously mentioned limitations, retirement of trained staff and lack of training of younger technicians werecited as important causes of potential closure of these facilities.

The purpose of this review is to assess how useful EM can be in diagnosis and reconfirmation of histopathological and immunofluorescence findings, using case examples to illustrate its importance in kidney biopsies.
CASE EXAMPLES (Corresponding to photomicrographs of electron microscopy)

1. Nephrotic range proteinuria with normal creatinine was seen in a 68 year-old male patient. Light microscopy was unremarkable and immunofluorescence showed a non-specific staining pattern. Electron microscopy was diagnostic in this case by showing the effacement of foot processes which is diagnostic for minimal change disease (fig.1).

2. A 51 year-old male patient presented with proteinuria, microscopic hematuria, slightly raised creatinine, positive ANA, and low c3 and C4. Though light microscopy and immunofluorescence showed typical patterns, the presence of tubuloreticular inclusions was re-assuring (fig.2).

3. A 33 year-old male patient presented with hematuria and nephrotic range proteinuria. Light microscopy showed features of lupus nephritis with endocapillary proliferation and wire loops. Immunofluorescence also showed a full house pattern. Electron microscopy was helpful in confirming that this was a mixed class IV with class V lupus nephritis (fig.3).

4. Increasing proteinuria was seen in a 35 year-old male patient. Light microscopy and immunofluorescence showed typical features of membranous glomerulonephritis, including diffuse thickening of glomerular basement membranes, spikes on silver stain, and $\mathrm{IgG}$ and $\mathrm{C} 3$ positivity. However, EM was needed in order to stage the disease as stage II to III (fig.4).

5. Transplanted 10 years back for Goodpasture's syndrome, a 38 year-old male presented with rising serum creatinine. Light microscopy showed double contours and glomerulitis and suggested the possibility of transplant glomerulopathy. Electron microscopy was diagnostic in this case by showing the presence of peritubularcapillary multilayering and it also reinforced the presence of double contours (fig.5). Hence, a diagnosis of transplant glomerulopathy was made.

6. A 62 year-old female presented with mild recurrent hematuria. Light microscopy was unremarkable and immunofluorescence was inconclusive as well, just showing nonspecific focal $\mathrm{c} 3$ staining. Electron microscopy showed marked thinning of glomerular basement membrane without which, a diagnosis of thin basement membrane disease would not have been possible (fig. 6).

7. A 38 years-old male presented with a slow rise in creatinine. Light microscopy and immunofluorescence were inconclusive. The presence of zebra bodies in the EM clinched the diagnosis of Fabry's disease.

Physical examination may be normal, but may reveal Electron microscopy, though not necessary in every biopsy, 


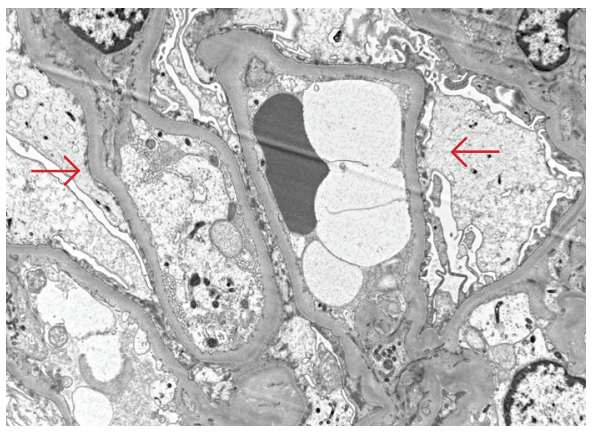

Figure 1: Swelling and effacement of foot processes is evident in podocytes in this case of minimal change disease (EM).

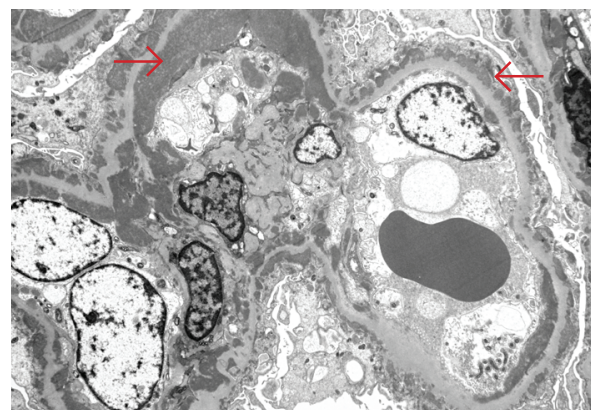

Figure 3: Subendothelial (wire loop) deposits and subepithelialdepoists, consistent with combined class IV and V lupus nephritis (EM).

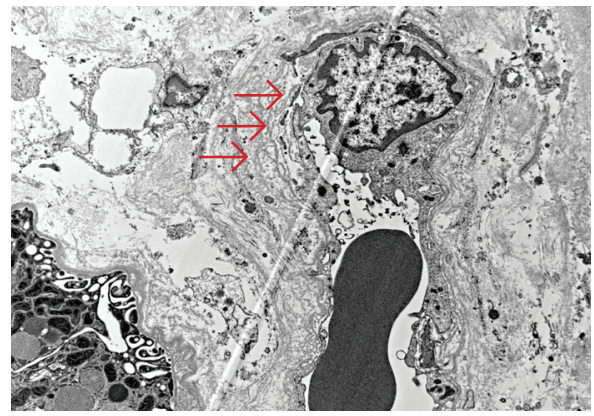

Figure 5: Transplant glomerulopathy case showing multilayering of the capillary basement membrane (arrows) (EM).

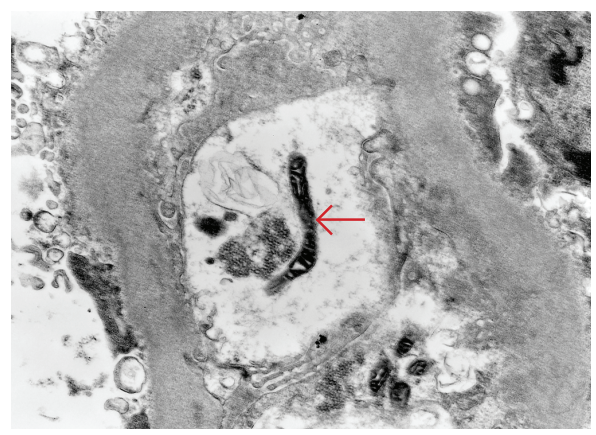

Figure 2: Tubuloreticular inclusions (arrow) are seen in the cytoplasm of this endothelial cell in this patient with lupus nephritis (EM).

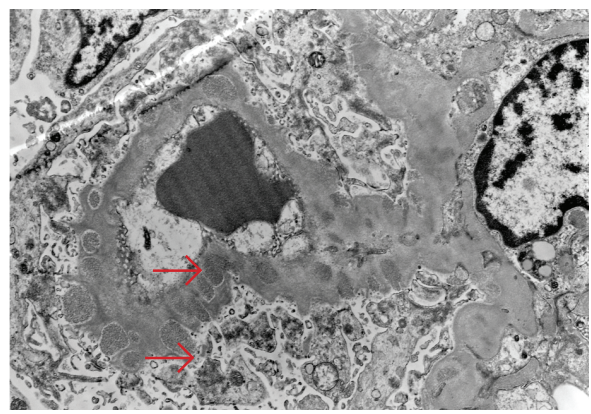

Figure 4: Intramembranous and subepithelial deposits in a patient with Membranous Glomerulonephritis Stage II to III (EM).

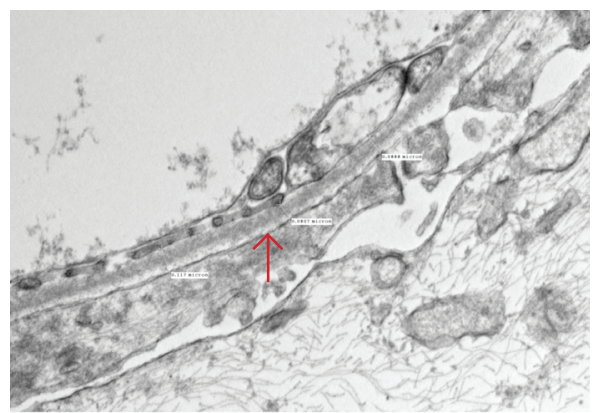

Figure 6: Thin Basement Membrane Disease showing thinning of basement membrane, measuring only 80-117 $\mathrm{nm}$ (EM).

Table 1: Contribution of Electron Microscopy to Renal Biopsy specimens as studied by various authors

\begin{tabular}{llcccc}
\hline Year & Author & Number of cases & $\begin{array}{c}\text { Essential for } \\
\text { Diagnosis }\end{array}$ & $\begin{array}{c}\text { Helpful diagnostic information } \\
\text { Not required for } \\
\text { diagnosis }\end{array}$ \\
\hline 1994 & Pearson & 88 & $25 \%$ & $50 \%$ & $25 \%$ \\
1996 & Haas & 233 & $21 \%$ & $21 \%$ & $58 \%$ \\
2005 & Collan & 111 & $18 \%$ & $54 \%$ & $28 \%$ \\
2007 & Wagrowska- Danilewicz & 113 & $31 \%$ & $13 \%$ & $56 \%$ \\
2011 & Mokhtar & 273 & $17 \%$ & $22 \%$ & $61 \%$ \\
2011 & Zuppan & 80 (pediatric cases) & $65 \%$ & $24 \%$ & $11 \%$ \\
\hline
\end{tabular}

is an investigation which may be required by the reporting pathologist if the diagnosis is not possible with routine and special stains and immunofluorescence; hence it is always advisable that some tissue be preserved for detailed evaluation if required. Some amount of tissue should be stored in glutaraldehyde; however formalin fixed tissue can also be used if required. ${ }^{9}$ 
Briefly, EM allows the assessment of cell proliferation (mesangialvs endothelial), changes in cell structure (podocyte foot process fusion/vacuolization), necrosis/ apoptosis, changes of glomerular and tubular basement membranes, localization of immune deposits, and specific morphologic findings in different diseases as detailed below. ${ }^{9}$

Different authors in their studies have evaluated the importance of EM categorized into three groups: essential for diagnosis, helpful for diagnosis and not required for diagnosis. The findings of these different studies are summarized in the table 1 .

In adult patients, it can be seen that up to $31 \%$ of the cases may require EM for diagnosis. On the average, about half the cases may not need EM for diagnosis, so it is not required that all samples should be subjected to that investigation. Which samples need to undergo EM should be left to the judgement of the pathologist.

Pearson et $\mathrm{al}^{2}$ noted in their study in 1994 that there wasn't any significant study done on EM in the last decade at the time of publication.A few years later in 1997, Haas published a similar important paper. ${ }^{5}$ In 2007, he reviewed his own paper retrospectively, in which he states that electron microscopy is necessary for diagnosis of the following lesions: $:^{10}$

- structural abnormalities of the glomerular basement membrane (GBM),such as Alport syndrome and thin GBM nephropathy;

- diseases with immune deposits characterized by an organizedsubstructure, such as fibrillary glomerulonephritis and $30-50 \%$ ofthose cases of cryoglobulinemic

- lysosomal storage diseases

- other forms of glomerulonephritis which are not diagnostic by light microscopy and immunofluorescence, e.g. postinfectious GN that is beyond the acute phase,dense deposit disease, and membranoproliferative glomerulonephritis type III

- minimal change nephropathy, because of its characteristic though not specific features on electron microscopy

- membranous nephropathy-particularly early lesions and latelesions, where there has been widespread resorption of immune complexdeposits and extensive glomerular basement membrane remodeling. It is also required for staging of membranous nephropathy.

- recurrent focal segmental glomerulonephritis in the early post-transplant period. It presents with proteinuria and effacement of foot processes by electron microscopy which precedes the appearance of light microscope findings by weeks to even months.

Ben-Bassat et $\mathrm{al}^{11}$ evaluated 37 cases of nephrotic syndrome and found electron microscopy to be of diagnostically useful in differentiating minimal change disease from early membranous glomerulonephritis.Mokhtaret $\mathrm{al}^{12}$ found that electron microscopy was most helpful for the confirmatory diagnosis of minimal change disease.

Haas further comments that electron microscopy can be helpful or even prognostic in other lesions like: ${ }^{10}$

- Lupus nephritis, especially mixed types

- Collapsing glomerulopathy, HIV (with the presence of tubuloreticular inclusions) vs non-HIV forms

- Latent diabetic nephropathy

- Membranous glomerulonephritis (secondary forms with mesangial deposits and tubuloreticular inclusions)

- Transplant glomerulopathy, especially early cases

Many pathologists may argue that IgA nephropathy can easily be diagnosed by light and immunofluorescence alone; however,EM can be essential in some cases, as demonstrated by Gu and Herrera. This is especially true in cases where deposits are rather subtle, focal or segmental. ${ }^{13}$

In 1997, Herrera et al ${ }^{14}$ published another paper in which they reviewed the importance of EM in transplant patients. This was seen to be the case in patients with transplant glomerulopathy, especially in early cases presenting mainly with subendothelial widening. Recurrence of glomerulonephritis in transplanted kidneys is another important area where EM can be very helpful; the immunosuppressive drugs may alter and subdue patterns of immunofluorescence. In chronic rejection, presence of lamellation of basement membranes in peritubular capillaries was another finding of importance noted at that time.

This finding of basement membrane multilayering was eventually incorporated into the Banff Criteria in 2005, as part of the diagnostic triad of late antibody mediated rejection. It was graded as moderate if there were 5-6 layers, and severe in cases having more than 7 layers, as seen under the electron microscope. ${ }^{15,16}$

Collan et al. $^{4}$ further demonstrated the importance of EM in transplant patients in whom it contributed to the final diagnosis in $86 \%$ of the studied patients. Its role in this study was to exclude the presence of recurrence of glomerulonephritis or amyloidosis. ${ }^{4}$ 
With all the above information and findings, it is quite apparent that electron microscopy still has a significant role to play in diagnosis of renal lesions. There is still a wide range of application of electron microscopy, especially in diagnosis of glomerulonephritis, in pediatric patients and in transplant patients.

\section{CONCLUSION}

Electron microscopy provides essential or helpful information in a substantial portion of renal biopsies, routine or transplant related. It helps determine the location of

\section{REFERENCES}

1. Jenette JC, Olson JL, Schwartz MM, Silva FG. Primer on the Pathologic Diagnosis of Renal Disease. In: Jennetts JC, Olson JL, Schwartz MM, Silva FG, eds. Hepinstall's Pathology of the Kidney. Lippincott Williams and Wilkins, 2007;98-123pp.

2. Pearson JM, McWilliam LJ, Coyne JD, Curry A. Value of electron microscopy in diagnosis of renal disease. J Clin Pathol 1994;47:1268.

3. Elhefnawy N. Contribution of Electron Microscopy to the Final Diagnosis of Renal Biopsies in Egyptian Patients. Pathol Oncol Res 2011;17:121-5.

4. Collan Y, Hirsimaki P, Aho H. Value of Electron Microscopy in Kidney Biopsy Diagnosis. Ultrastructural Pathol 2005;29:461-8.

5. Haas M. A revaluation of routine electron microscopy in the examination of native renal biopsies. J Am Soc Nephrol 1996;8:70-6.

6. Wagrowska-Danilewicz M, Danilewicz M. Current position of electron microscopy in the diagnosis of glomerular diseases. Pol J Pathol 2007;58:87-92.

7. Zuppan C. Role of Electron Microscopy in the Diagnosis of Nonneoplastic Renal Disease in Children.Ultrastructural Pathol 2011;35:240-4.

8. De Haro T, Furness P. Current and Future Delivery of Diagnostic Electron Microscopy in the UK: results of a national survey. J Clin Pathol 2012;65:357-61. deposits, detects very small deposits, and helps find alteration of cellular and basement membrane structure. Electron microscopy has been shown to be essential for diagnosis in up to $31 \%$ of biopsies - these caseswere either initially in conclusive or they had an altered diagnosis after EM was performed on the tissue of interest. Thus, it still remains an important tool for diagnosis of glomerular diseases, without which diagnostic accuracy may be compromised. Despite its high initial and maintenance costs, it is an investigation that needs to be part of a diagnostic facility in a big hospital which is regularly performing renal biopsies.

9. Amann K, Haas CS. What You Should Know About the Work-up of a Renal Biopsy. Nephrol Dial Transplant 2006;21:1157-61.

10. Haas M:Electron Microscopy in Renal Biopsy Interpretation - When and Why We Still Need It. US Renal Dis 2007;pp 20-2.

11. Ben-Bassat M, Stark H, Robson M, Rosenfield J. Value of routine electron microscopy in the differential diagnosis of nephrotic syndrome. Pathol Microbiol 1974;41:26-40.

12. Ghadeer AM, Sawsan MJ. Role of Electron Microscopy in Evaluation of Native Kidney Biopsy; A Retrospective Study of 273 Cases. Iran J Kidney Dis 2011;5:314-9.

13. Gu X, Herrera GA. The value of electron microscopy in the diagnosis of IgA nephropathy. Ultrastructural Pathol 2002;26:203-10.

14. Herrera GA, Isaac J, Turbat-Herrera EA. Role of Electron Microscopy in Transplant Renal Pathology. Ultrastructural Pathol 1997;26:481-98.

15. Racusen LC, Solez K, Colvin RBet al. The Banff 97 working classification of renal allograft pathology. Kidney Int 1999;55:71320 .

16. Solez K, Racusen LC, Sis B, et al. Banff '05 Meeting Report: Differential Diagnosis of Chronic Allograft Injury and Elimination of Chronic Allograft Nephropathy. Am J Transplant 2007;7:518-26. 